\title{
Elucidation of Nucleic Acid-Drug Interactions by Tandem Mass Spectrometry
}

\author{
Yvonne Hari, Adrien Nyakas, Silvan R. Stucki, and Stefan Schürch*
}

\begin{abstract}
In continuation of the long tradition of mass spectrometric research at the University of Bern, our group focuses on the characterization of nucleic acids as therapeutic agents and as drug targets. This article provides a short overview of our recent work on platinated single-stranded and higher-order nucleic acids. Nearly three decades ago the development of soft ionization techniques opened a whole new chapter in the mass spectrometric analysis of not only nucleic acids themselves, but also their interactions with potential drug candidates. In contrast to modern next generation sequencing approaches, though, the goal of the tandem mass spectrometric investigation of nucleic acids is by no means the complete sequencing of genetic DNA, but rather the characterization of short therapeutic and regulatory oligonucleotides and the elucidation of nucleic acid-drug interactions. The influence of cisplatin binding on the gas-phase dissociation of nucleic acids was studied by the means of electrospray ionization tandem mass spectrometry. Experiments on native and modified DNA and RNA oligomers confirmed guanine base pairs as the preferred platination site and laid the basis for the formulation of a gas-phase fragmentation mechanism of platinated oligonucleotides. The study was extended to doublestranded DNA and DNA quadruplexes. While duplexes are believed to be the main target of cisplatin in vivo, the recently discovered DNA quadruplexes constitute another promising target for anti-tumor drugs owing to their regulatory functions in the cell cycle.
\end{abstract}

Keywords: Cisplatin · Gas-phase dissociation · Higher-order nucleic acids · Nucleic acid-drug interactions . Tandem mass spectrometry

\section{Oligonucleotides in Human Therapy}

Nucleic acids play a crucial role in cellular processes, as they are key elements responsible for storage and replication of the genetic information and its conversion into proteins. Moreover, they perform manifold regulatory and catalytic activities. Consequently, it is not surprising that nucleic acids have gained increased attention as targets for the treatment of generelated diseases. Among DNA-targeting drugs, cis-dichlorodiammineplatinum(II), known as cisplatin, was the first transition metal-based compound to be introduced as an anti-tumor agent and it has been administered for the successful treatment of various types of cancer to this day. Cisplatin is known to bind preferentially to adjacent guanine bases of deoxyribonucleic ac-

\footnotetext{
*Correspondence: PD Dr. S. Schürch

Department of Chemistry and Biochemistry

University of Bern

Freiestrasse 3

$\mathrm{CH}-3012$ Bern

Tel.: +41316314389

E-mail: stefan.schuerch@dcb.unibe.ch
}

ids (DNA), thus creating 1,2-intrastrand cross-links. This causes the DNA duplex to bend and consequently inhibits DNA replication and transcription. Unfortunately, the therapy provokes severe side effects, which triggered the search for alternative agents. Second- and third-generation platinum-based chemotherapeutics aim at increased selectivity of the drugs as well as improved delivery to their targets. While compounds based on substitute transition metals, namely ruthenium, are currently being evaluated for their anti-proliferative activity against tumor cells as well, platinum-based agents remain unique in that their principal target in the cell is DNA.

\section{Tandem Mass Spectrometry of Oligonucleotides}

The development of drugs targeting nucleic acids necessitates suitable analytical tools for the elucidation of DNA-drug interactions as well as for the characterization of synthetic oligonucleotide analogues. Technological progress in the past decades, in particular the introduction of soft ionization techniques and the availability of highly accurate mass analyzers, rendered tandem mass spectrometry a powerful instrument to address these tasks. Information on nucleotide sequences, the types and positions of modifications, and the binding patterns of drugs is provided by characteristic fragment ions.

However, a detailed understanding of the gas-phase fragmentation of nucleic acids is a mandatory prerequisite for unambiguous data interpretation. The major dissociation pathways of oligonucleotides are the loss of nucleobases and cleavage of the backbone at the phosphate groups. The 5'-terminal backbone fragment ions, which arise from the scission of one of the four phosphodiester bonds, are commonly referred to as a-, b-, c-, and d-ions, whereas the series of complementary 3'-terminal backbone fragments are termed w-, x-, y-, and z-ions (Fig. 1). ${ }^{[1]}$

Even minor modifications of the phosphate group or the sugar moiety, such as substitution of the 2'-H in DNA by the hydroxyl group in RNA, result in significantly altered fragmentation pathways. Upon collisional activation, DNA mainly generates complementary (a-B)-/w-ion pairs due to cleavage of the 3'-C-O bond in conjunction with the loss of a neutral nucleobase. ${ }^{[2,3]} \mathrm{On}$ the other hand, the gas-phase dissociation of RNA is directed by the additional 2'-hydroxyl group. Abstraction of the 2'-proton and subsequent formation of a cyclic intramolecular transition state induce backbone cleavage at the 5'-C-O bond, thus forming the corresponding c-/y-ion pair.[4,5] Within 


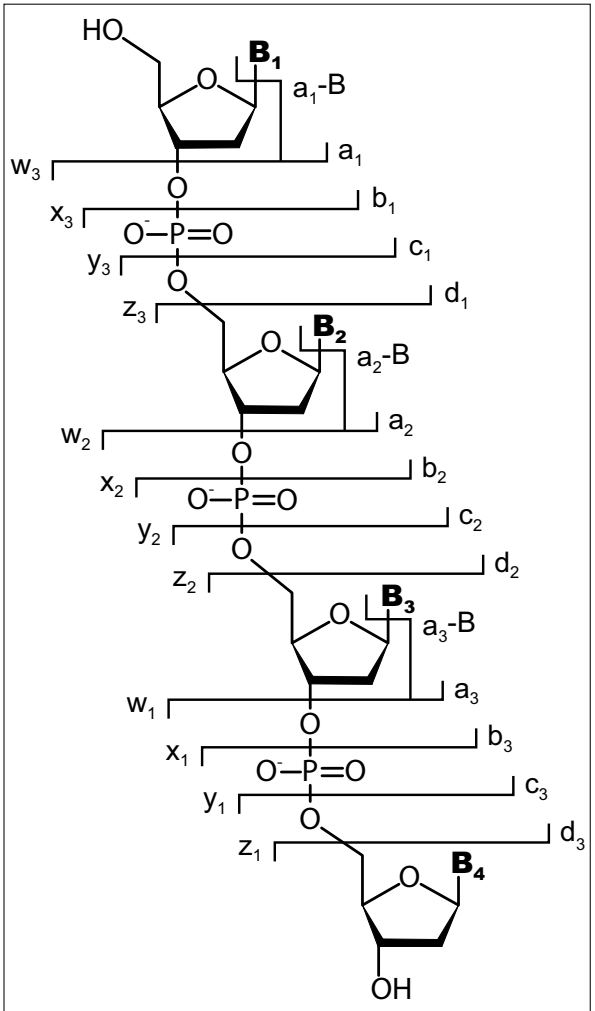

Fig. 1. McLuckey nomenclature for oligonucleotide fragment ions. ${ }^{[1]}$

recent years, a comprehensive armamentarium of dissociation mechanisms has been assembled, which can be applied to deciphering tandem mass spectrometric data obtained from DNA, RNA, and the many structurally altered oligonucleotide analogues. [6-12]

\section{Interaction of Cisplatin with Single-stranded Nucleic Acids}

Mass spectrometry has been widely applied to demonstrate the formation of adducts between nucleic acids and transition metal-based anti-cancer drugs. ${ }^{[13-15]}$ However, very scarce information is found in literature about the gas-phase fragmentation of these adducts. In recent years, a couple of fundamental studies started to shed light on the influence of adduct formation on the gas-phase dissociation mechanisms of nucleic acids. ${ }^{[16,17]}$

\subsection{Single-stranded DNA}

Tandem mass spectrometric investigation of DNA-cisplatin adducts gave evidence for the cisplatin moiety altering the dissociation behavior of oligonucleotides. ${ }^{[16]}$ When subjecting platinated hexamers containing a vicinal GG-pair to collision-induced dissociation (CID), partial loss of the ammine ligands was observed along with the formation of unplatinated as well as platinated backbone fragments. The latter always comprise at least one of the guanines, which singles out this purine nucleobase as the target of cisplatin-binding. The most striking characteristic of the product ion spectra of platinated oligonucleotides is the increased intensity of a sequence specific $\mathrm{w}$-ion, which is generated by cleavage of the 3'-C-O bond adjacent to the GG-pair, thus substantiating this base pair as the preferred platination site (Fig. 2).

The increased cleavage of the 3'-C-O bond as a result of platination can be rationalized based on the most prevalent gas-phase dissociation pathway of DNA initialized by the protonation of a nucleobase, which leads to the formation of the DNA-typical (a-B)-/w-ion pair. Like protonation, the positively charged platinum moiety destabilizes the N-glycosidic bond of the platinated base, thus enhancing backbone cleavage at the 3'-phosphate of the respective nucleotide. ${ }^{[16]}$

5 '-terminal fragments complementary to the high-intensity w-ion include the $(\mathrm{a}+\mathrm{Pt})$-ion as well as the corresponding (unplatinated) (a-B)-ion. Co-occurrence of these ions provides evidence for multiple dissociation pathways. The proposed fragmentation pathways were further substantiated by tandem mass spectrometric experiments of platinated methylphosphonate DNA. While methylphosphonate nucleic acids themselves are of interest for antisense applications, they represent a valuable instrument to study the role of specific phosphate groups in gas-phase

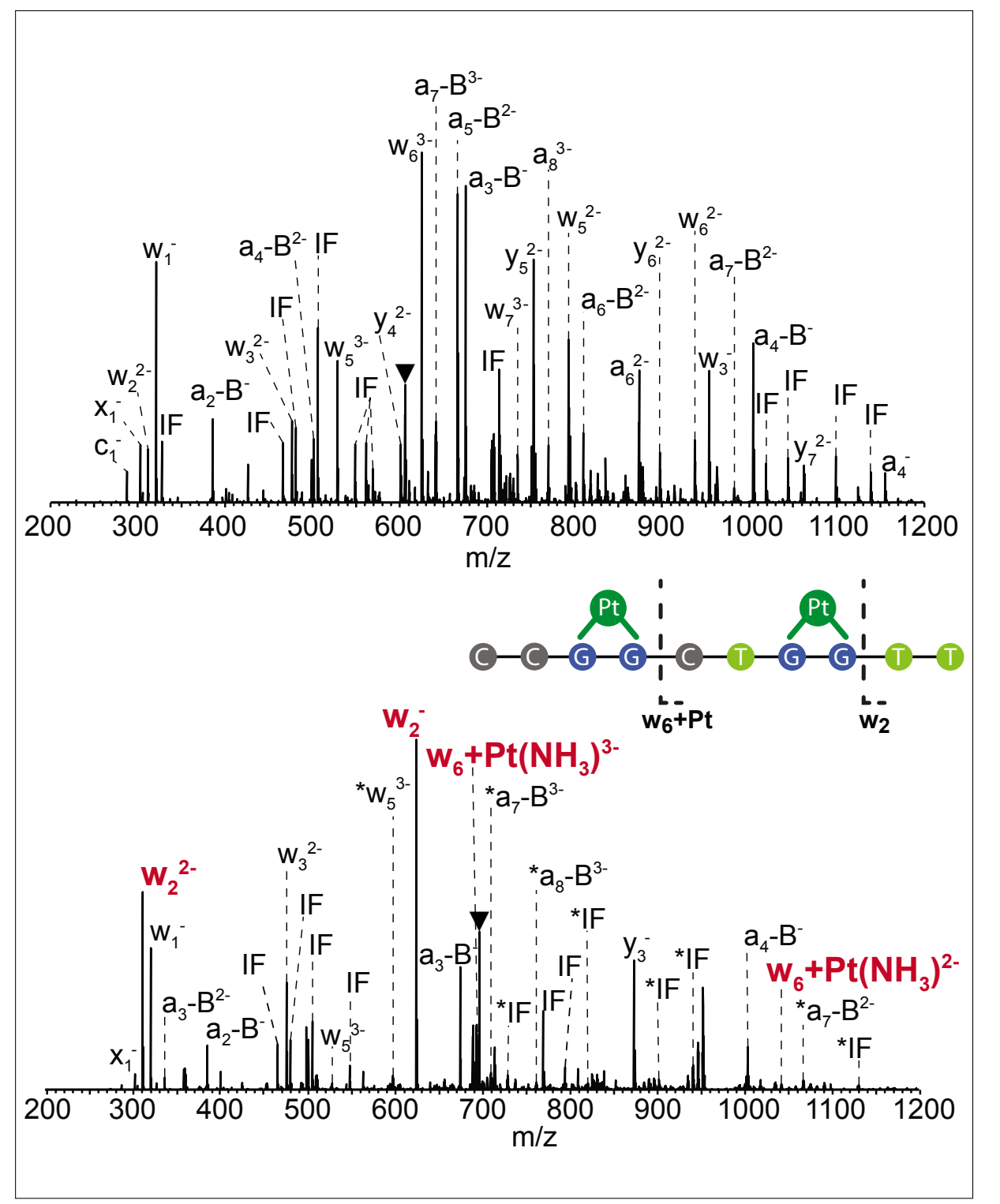

Fig. 2. Effect of platination on the fragment ion pattern of the single-stranded decadeoxynucleotide d(CCGGCTGGTT). Top: Product ion spectrum of the unplatinated oligonucleotide obtained by collision-induced dissociation of the five-fold negatively charged precursor ion. Bottom: Product ion spectrum of the five-fold negatively charged doubly platinated decamer. Platination promotes cleavage of the 3 '-C-O bonds adjacent to the adduct sites, resulting in a very abundant $\mathrm{w}_{2}$-ion and a platinated $w_{6}$-ion, indicating that $G_{3} G_{4}$ and $G_{7} G_{8}$ are preferred platination sites. $(\nabla=$ precursor, ${ }^{*}=$ platinated fragment, IF $=$ internal fragment). 
dissociation mechanisms, since methylation of backbone phosphates precludes deprotonation, thus masking potentially reactive sites. ${ }^{[16]}$

\subsection{Single-stranded RNA}

Remarkably, product ion spectra of platinated oligoribonucleotide hexamers exhibit an increase of the same sequence specific w-ions. Such increase was unexpected, because the predominant fragments occurring upon dissociation of RNA are $\mathrm{c}$ - and y-ions. Increased cleavage of the 3'-C-O bond adjacent to the GG-pair as a consequence of platination distinguishes the guanine bases as most probable binding site. Comparative analysis of 2'-O-methyl oligoribonucleotides confirmed that the 2'-hydroxyl group is not involved in the formation of the high-intensity w-ion. ${ }^{[9]}$ In fact, the platinated RNA dissociates in a DNA-like manner as a result of the cisplatin moiety that destabilizes the $\mathrm{N}$-glycosidic bond.

\section{Interaction of Cisplatin with Higher-Order Nucleic Acid Structures}

Higher-order nucleic acid structures and their adducts formed with covalently binding and even intercalating agents are preserved during transfer into the gasphase by electrospray ionization and thus, can be subjected to tandem mass spectrometric investigation. The resulting product ion spectra permit the adduct sites to be accurately pinpointed and reflect the influence of the drugs on the gas-phase stability of the structures.

\subsection{Duplex-DNA}

In vivo complementary nucleic acids assume double-helical structures. Distortion of these well-ordered assemblies is an important mechanism of the anti-tumor activity of cisplatin and related chemotherapeutic agents. The preferred platination site observed for linear oligonucleotides extends to double-stranded DNA and the presence of a cisplatin moiety appears to destabilize the native DNA duplex in the gas-phase. Consequently, the transfer of the intact platinated duplex into the gasphase is very delicate.

Our group successfully isolated an intact platinated DNA duplex and subjected it to CID for the first time. ${ }^{[18]}$ While fragmentation of duplexes is generally characterized by strand separation, increased formation of terminal and internal backbone fragments were found to complement strand separation of platinated duplexes (Fig. 3). This result indicates that platination promotes the cleavage of covalent bonds, while the non-covalent interactions

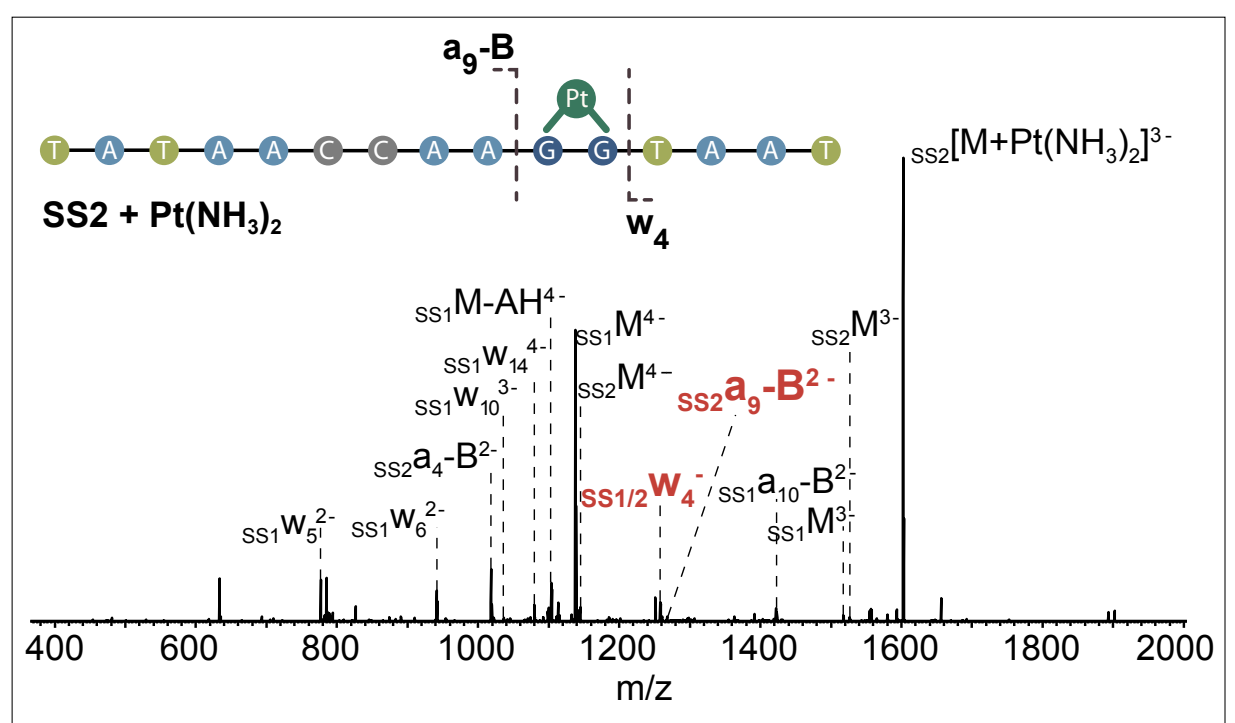

Fig. 3. Product ion spectrum of platinated duplex DNA. The most abundant peaks correspond to the two single strands SS1 5'-ATTACCTTGGTTATA-3' and SS2 5'-TATAACCAAGGTAAT-3'. Occurrence of the $w_{4}^{-}$- and an unplatinated $\left(a_{9}-B\right)^{2-}$-ion give evidence for $G_{10} G_{11}$ of $S S 2$ as the platination site. The inset shows the platinated single strand SS2 of the duplex.

between the two single strands remain preserved.

Both single strands were detected in unplatinated and in their platinated form, giving evidence for platination occurring at different positions. The relative peak intensities, however, confirm that the cisplatin moiety is preferentially attached to SS2. As indicated in Fig. 3, formation of the $\mathrm{w}_{4}^{--}$and $\left(\mathrm{a}_{9}-\mathrm{B}\right)^{2-}$-ion point towards the vicinal GG-pair of SS2 as the most probable platination site. By subjecting the platinated SS2 to an additional stage of collisional activation in an $\mathrm{MS}^{3}$ experiment, cleavage of the 3'-C-O bond adjacent to $\mathrm{G}_{11}$, was identified as the major dissociation pathway, thus, implying that platination occurred at $G_{10} G_{11}$ of SS2.

\subsection{Quadruplex-DNA}

Beside the double helix, which represents the most common in vivo structure of nucleic acids, alternative higher-order structures such as DNA quadruplexes constitute potential targets for anti-cancer drugs as well. The structural basis of DNA quadruplexes is the G-tetrad, a planar arrangement of four guanine bases connected via Hoogsteen hydrogen bonding, which is further stabilized by the incorporation of monovalent cations. Scientific interest in the structure arose from the discovery that the repetitive G-rich human telomere sequence (TTAGGG) forms quadruplexes in vitro. ${ }^{[19-22]}$ Although DNA quadruplexes elude direct detection in vivo, antibody labelling and the discovery of quadruplexbinding proteins gave substantial evidence for their existence, and even more important, their regulatory function in living organisms. ${ }^{[23,24]}$ The proposed involvement of quadruplex DNA in cell cycle control and chromosome organization makes them promising drug targets and motivated the elucidation of their interaction with anticancer drugs.

Electrospray mass spectrometry was demonstrated by several research groups to be a suitable tool for exploring the in vitro formation of tetra-, bi-, and monomolecular quadruplexes ${ }^{[25-28]}$ and even mechanistic data were provided about the gas-phase dissociation of these intriguing higher-order structures. ${ }^{[15,26,29]}$

In a recent study, we reported on the investigation of the dissociation behavior of quadruplex DNA and cisplatin adducts thereof by high-resolution electrospray tandem mass spectrometry. ${ }^{[29]}$ The (unplatinated) tetramolecular assembly $\left[\mathrm{d}\left(\mathrm{TG}_{6} \mathrm{~T}\right)_{4}\right.$ $+5 \mathrm{NH}_{4}^{+}$] (see inset in Fig. 4) served as a first model compound laying the basis for the detailed characterization of the interaction of transition metal-based anti-cancer drugs with quadruplex DNA. A major dissociation pathway of the six-fold negatively charged quadruplex is the loss of its incorporated ammonium ions as well as multiple neutral guanine bases. Moreover, the discovery of truncated quadruplex ions (TQIs) gives evidence for the ejection of terminal backbone fragments from the intact quadruplex, which further reflects the extraordinarily high gas-phase stability of tetramolecular quadruplexes. The third characteristic fragmentation channel is the disassembly of the quadruplex into a single strand and a triplex structure.

As shown in Fig. 4, CID of the platinated tetramolecular quadruplex essentially generates the same types of fragment ions. The cisplatin moiety, however, was found to be exclusively located on the TQIs and the triplex, while no platinated single 


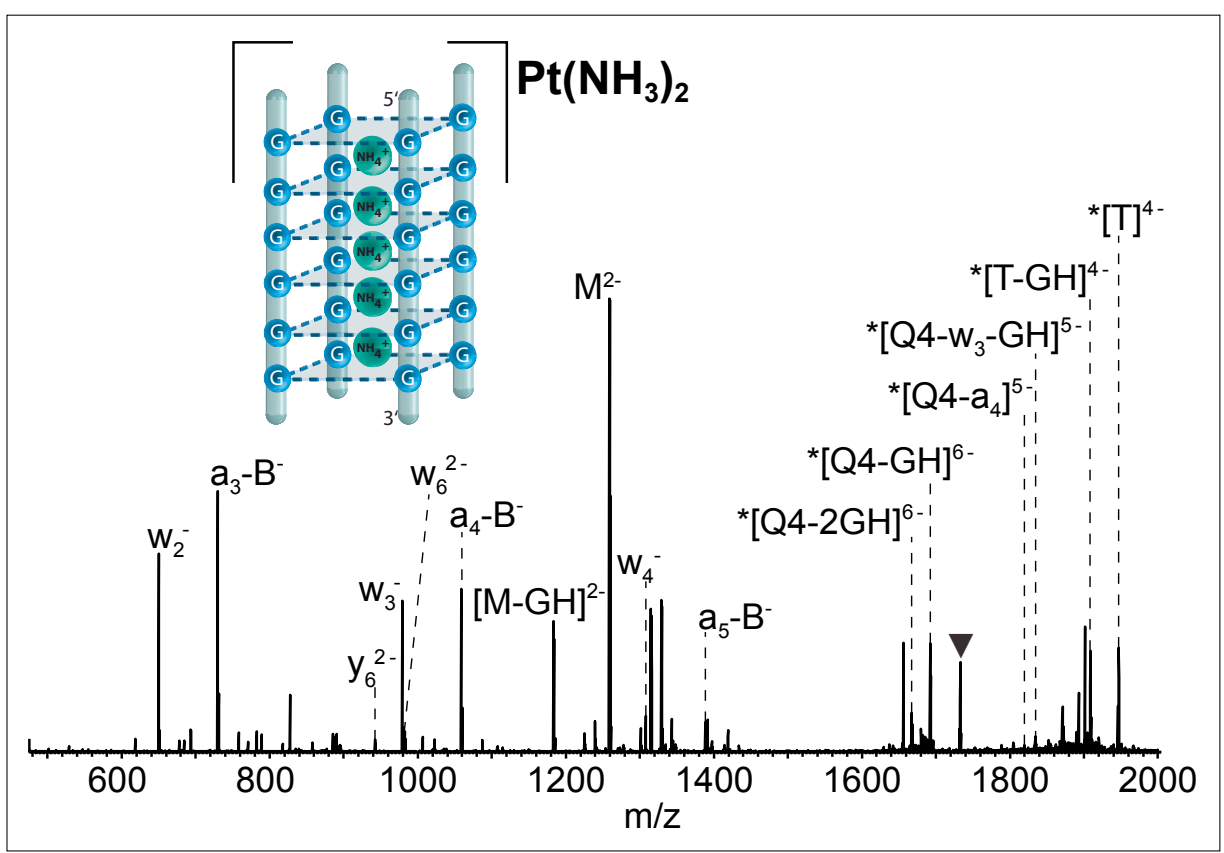

Fig. 4. Product ion spectrum of a platinated tetramolecular DNA quadruplex. CID of the platinated quadruplex is characterized by the ejection of terminal backbone fragments and unplatinated single strands, giving rise to platinated truncated quadruplex and triplex structures $(\boldsymbol{\nabla}=$ precursor, ${ }^{*}=$ platinated fragments). The inset shows the structure of the tetramolecular quadruplex with five embedded ammonium ions.

strands nor platinated backbone fragments were detected. The ejection of unplatinated fragments and unweaving of an unplatinated single strand are clearly favored.

By extending the study to bimolecular and monomolecular quadruplexes with the G-repeats connected covalently by singlestranded loop regions, we could demonstrate that the loop regions represent the most likely platination sites. The gas-phase dissociation of bimolecular quadruplexes is characterized by strand separation and simultaneous minor backbone cleavage. Tandem mass spectrometric experiments of platinated heterobimolecular quadruplexes indicate, that interstrand cross-links arise from cisplatin binding to purine bases in the loop region. In case of the monomolecular quadruplex the results are more ambiguous. Tandem mass spectrometric characterization of the quadruplex-forming human telomeric sequence (TTAGGG) demonstrates increased resistance to backbone cleavage compared to the unannealed oligonucleotide, thus giving evidence for the presence of the platinated higher-order structure. Data implies that the most likely platination sites are located at the termini of the sequence.

\section{Conclusions}

Though still in its infancy, tandem mass spectrometry of nucleic acid-drug adducts harbors a great potential in the field of gene-related diseases. Starting from the sequencing of natural and modified nucleic acids, the focus of study has been extended to the gas-phase dissociation of higher-order structures including DNA duplexes and quadruplexes. Furthermore, the presented experiments promote mass spectrometry as a means of characterizing the interaction of oligonucleotides with transition metal-based drugs such as cisplatin, which will broaden our understanding of the mode of action of these therapeutics.

\section{Acknowledgement}

The authors would like to thank the Swiss National Science Foundation for the generous financial support of this work.

[1] S. A. McLuckey, G. J. Van Berkel, G. L. Glish, J. Am. Soc. Mass. Spectrom. 1992, 3, 60.

[2] S. Pan, K. Verhoeven, J. K. Lee, J. Am. Soc. Mass. Spectrom. 2005, 16, 1853.

[3] Z. Wang, K. X. Wan, R. Ramanathan, J. S. Taylor, M. L. Gross, J. Am. Soc. Mass. Spectrom. 1998, 9, 683

[4] T. Y. Huang, A. Kharlamova, J. Liu, S. A. McLuckey, J. Am. Soc. Mass. Spectrom. 2008, $19,1832$.

[5] J. M. Tromp, S. Schürch, J. Am. Soc. Mass. Spectrom. 2005, 16, 1262.

[6] Y. Gao, S. A. McLuckey, J. Mass Spectrom. 2012, 47, 364.

[7] T. Y. Huang, A. Kharlamova, S. A. McLuckey, J. Am. Soc. Mass. Spectrom. 2010, 21, 144.

[8] S. T. Monn, S. Schürch, J. Am. Soc. Mass. Spectrom. 2007, 18, 984.

[9] A. Nyakas, S. R. Stucki, S. Schürch, J. Am. Soc. Mass. Spectrom. 2011, 22, 875.

[10] S. I. Smith, J. S. Brodbelt, Anal. Chem. 2011, $83,303$.

[11] N. Tretyakova, P. W. Villalta, S. Kotapati, Chem. Rev. 2013, 113, 2395.

[12] J. Wu, S. A. McLuckey, Int. J. Mass Spectrom. 2004, 237, 197.

[13] J. S. Brodbelt, Annu. Rev. Anal. Chem. 2010, 3, 67.

[14] M. Groessl, Y. O. Tsybin, C. G. Hartinger, B. K. Keppler, P. J. Dyson, J. Biol. Inorg. Chem. 2010, $15,677$.

[15] C. Silvestri, J. S. Brodbelt, Mass Spectrom. Rev. 2013, 32, 247.

[16] A. Nyakas, M. Eymann, S. Schürch, J. Am. Soc. Mass. Spectrom. 2009, 20, 792

[17] Z. Xu, J. B. Shaw, J. S. Brodbelt, J. Am. Soc. Mass. Spectrom. 2013, 24, 265.

[18] A. Nyakas, L. C. Blum, S. R. Stucki, J. L. Reymond, S. Schürch, J. Am. Soc. Mass. Spectrom. 2013, 24, 249.

[19] E. H. Blackburn, J. G. Gall, J. Mol. Biol. 1978, $120,33$.

[20] S. Haider, G. N. Parkinson, S. Neidle, J. Mol. Biol. 2002, 320, 189.

[21] R. K. Moyzis, J. M. Buckingham, L. S. Cram, M. Dani, L. L. Deaven, M. D. Jones, J. Meyne, R. L. Ratliff, J. R. Wu, Proc. Natl. Acad. Sci. USA 1988, 85, 6622 .

[22] M. Gellert, M. N. Lipsett, D. R. Davies, Proc. Natl. Acad. Sci. USA 1962, 48, 2013.

[23] H. J. Lipps, D. Rhodes, Trends Cell Biol. 2009, 19,414

[24] C. Schaffitzel, I. Berger, J. Postberg, J. Hanes, H. J. Lipps, A. Pluckthun, Proc. Natl. Acad. Sci. USA 2001, 98, 8572

[25] X. H. Guo, S. Y. Liu, Z. Yu, J. Am. Soc. Mass. Spectrom. 2007, 18, 1467.

[26] C. L. Mazzitelli, J. Wang, S. I. Smith, J. S. Brodbelt, J. Am. Soc. Mass. Spectrom. 2007, 18, 1760.

[27] F. Rosu, V. Gabelica, C. Houssier, P. Colson, E. De Pauw, Rapid Commun. Mass Spectrom. 2002, 16, 1729.

[28] G. Yuan, Q. Zhang, J. Zhou, H. H. Li, Mass Spectrom. Rev. 2011, 30, 1121.

[29] S. R. Stucki, A. Nyakas, S. Schürch, J. Mass Spectrom. 2011, 46, 1288. 